\title{
Metabolism of Procainamide to the Cytotoxic Hydroxylamine by Neutrophils Activated In Vitro
}

\author{
Robert L. Rubin and John T. Curnutte \\ Department of Basic and Clinical Research, Research Institute of Scripps Clinic, La Jolla, California 92037
}

\begin{abstract}
An almost universal side effect of long-term therapy with procainamide is the appearance of serum autoantibodies and less frequently a syndrome resembling lupus erythematosus. Previous studies demonstrated that procainamide-hydroxylamine (PAHA), a metabolite generated by hepatic mixed function oxidases, was highly toxic to dividing cells, but evidence that PAHA could be formed in the circulation was lacking. This study examines the capacity of neutrophils to metabolize procainamide to reactive forms. Neutrophils activated with opsonized zymosan were cytotoxic only if procainamide was present, whereas $\boldsymbol{N}$-acetyl procainamide, which does not induce autoimmunity, was inert in this bioassay. PAHA was detected by HPLC in the extracellular medium if ascorbic acid was present. Generation of PAHA and cytotoxic procainamide metabolites was inhibited by $\mathrm{NaN}_{3}$ and catalase but not by superoxide dismutase, indicating that $\mathrm{H}_{2} \mathrm{O}_{2}$ and myeloperoxidase were involved. Nonactivated neutrophils and neutrophils from patients with chronic granulomatous disease did not generate cytotoxic PAHA, demonstrating that $\mathrm{H}_{2} \mathrm{O}_{2}$ was derived from the respiratory burst accompanying neutrophil activation. These conclusions were supported by results of a cell-free system in which neutrophils were replaced by myeloperoxidase and $\mathrm{H}_{2} \mathrm{O}_{2}$ or an $\mathrm{H}_{2} \mathrm{O}_{2}$ generating system. These studies demonstrate the capacity of neutrophils to mediate metabolism of procainamide and establish the role of myeloperoxidase released during degranulation and $\mathrm{H}_{2} \mathrm{O}_{2}$ derived from the respiratory burst in the direct cooxidation of procainamide to PAHA. The profound biologic activity of this metabolite and its possible generation within lymphoid compartments implicate this process in the induction of autoimmunity by procainamide.
\end{abstract}

\section{Introduction}

Antinuclear antibodies commonly arise during long-term treatment with procainamide, and $15-20 \%$ of these patients develop a lupus-like illness $(1,2)$. The mechanism for procainamide-induced autoimmunity has not been established, but various hypotheses have been explored including allergiclike hypersensitivity reactions, polyclonal activation, and perturbation of lymphocyte recognition processes.

Address reprint requests to Dr. Rubin, Department of Basic and Clinical Research (BCR6), Research Institute of Scripps Clinic, 10666 North Torrey Pines Road, La Jolla, CA 92037.

Received for publication 30 August 1988 and in revised form 29 November 1988.

J. Clin. Invest.

(C) The American Society for Clinical Investigation, Inc.

$0021-9738 / 89 / 04 / 1336 / 08 \quad \$ 2.00$

Volume 83, April 1989, 1336-1343
Procainamide contains an aromatic amino group that is acetylated in vivo by hepatic acetyl transferases. The genetically determined slow acetylator phenotype is associated with more rapid onset of autoantibodies and symptomatic drug-induced lupus compared to rapid acetylators (3), and the $N$-acetylated derivative of procainamide fails to induce autoantibodies and symptoms of lupus $(4,5)$. These observations were interpreted to suggest that the free amino group on procainamide participates directly in the induction of autoimmunity and stimulated numerous attempts to demonstrate in vitro effects of procainamide on lymphocyte function and/or affinity for macromolecules. Conflicting results (6-11) and even immunosuppressive effects $(6,9,12)$ have been reported in studies using pharmacologically realistic procainamide concentrations, raising doubts about a direct role for procainamide in autoimmunity induction.

An alternative explanation for the inability of $\mathrm{N}$-acetyl procainamide to induce autoimmunity is that metabolism of procainamide near the aromatic amine moiety generates the active, autoimmunity inducing compound, and acetylation blocks this transformation. We recently demonstrated that one procainamide analogue, procainamide-hydroxylamine (PAHA), ${ }^{1}$ is highly cytotoxic to many cell types in vitro, killing rapidly dividing cells including activated lymphocytes at concentrations well within the in vivo therapeutic concentration of procainamide (13). However, previous studies showed procainamide metabolism to the hydroxylamine only by the microsomal fraction from rat and human liver homogenates containing an NADPH generating system $(14,15)$. Since PAHA is highly reactive, rapidly binding to microsomal proteins (16) and losing its cytotoxic potency in high serum concentrations (13), the potential for liver generated PAHA to react with extrahepatic lymphoid tissue appeared unlikely. Presence of PAHA within a lymphoid compartment would seem to be a prerequisite to implicate it in the initiation or maintenance of immune dysfunction leading to autoimmunity.

Our preliminary results suggested that peripheral blood neutrophils may mediate oxidation of procainamide to PAHA (17), and this has been confirmed (18). By application of a bioassay for cytotoxic procainamide metabolites as well as direct chemical analyses, the current study demonstrates the capacity of activated neutrophils to metabolize procainamide to the cytotoxic hydroxylamine. These results provide the precedent and delineate a mechanism for an immunologically rele-

1. Abbreviations used in this paper: CGD, chronic granulomatous disease; HX, hypoxanthine; MPO, myeloperoxidase; NAPA, $N$-acetyl procainamide; nitro-PA, nitro-procainamide (4-nitro- $N$-diethylaminoethylbenzamide); PA, procainamide (4-amino- $N$-diethylaminoethylbenzamide); PMA, phorbol 12-myristate 13-acetate; XO, xanthine oxidase. 
vant transformation of procainamide to a potentially pathogenic form.

\section{Methods}

Human donors. Normal neutrophils were obtained from healthy donors who avoided anti-inflammatory drugs including aspirin for one week before blood donation. Neutrophils with abnormal respiratory burst capacities were obtained from patients with chronic granulomatous disease (CGD). One patient was a 19-yr-old male with the classic, $X$-linked/cytochrome $b_{558}$-negative form of CGD (19). Neutrophils from this patient contained no detectable cytochrome $b_{558}$ by spectrophotometric (19) or Western blot analyses (20), and showed no respiratory burst activity when stimulated with either phorbol 12-myristate 13-acetate (PMA) or opsonized zymosan (see Results). Another patient was a 35-yr-old female with the rare autosomal recessive/cytochrome $b_{558}$-negative form of CGD (20-23). Her cells showed a negligible respiratory burst in response to PMA (see Results).

Preparation of neutrophils. Neutrophils were isolated from blood anticoagulated with $0.057 \mathrm{M}$ acidic citrate buffer containing $0.037 \mathrm{M}$ dextrose based on methodologies previously described $(19,24)$. Briefly, erythrocytes were removed by $1 g$ sedimentation through $2 \%$ dextran and by rapid lysis of the leukocyte containing supernatant with ice cold water, twice. Platelets were removed by repeated washing of pelleted leukocytes in PBS $(0.01 \mathrm{M}$ phosphate buffer, $0.14 \mathrm{M} \mathrm{NaCl}, \mathrm{pH} 7.4$ in deionized water) at $4^{\circ} \mathrm{C}$, and neutrophils were separated from mononuclear cells by sedimentation through a Ficoll-Hypaque step gradient. The neutrophil pellet was washed twice in PBS, $4^{\circ} \mathrm{C}$. Viability by trypan blue dye exclusion and purity were routinely $>97 \%$. Cell numbers were determined by hemocytometer count, and the preparation was stored on ice for use within a few hours.

Neutrophil function assays. Respiratory burst was measured by generation of superoxide anion $\left(\mathrm{O}_{2}^{-}\right)$based on Johnston and Lehmeyer (25), and Van Epps et al. (26). Neutrophils were suspended in Dulbecco's PBS (ICN Radiochemicals, Irvine, CA) containing $1 \mathrm{mg} / \mathrm{ml}$ glucose, and $0.08 \mathrm{mM}(1.0 \mathrm{mg} / \mathrm{ml})$ cytochrome $c$ (Sigma Chemical Co., St. Louis, MO, type III). Boiled zymosan (type A, Sigma Chemical Co.) was washed in PBS and incubated with human serum (fresh or stored at $-70^{\circ} \mathrm{C}$ ) for $0.5 \mathrm{~h}, 37^{\circ} \mathrm{C}$ at a ratio of $80 \mathrm{mg}$ zymosan $/ 10 \mathrm{ml}$ serum. The opsonized zymosan was washed twice in PBS by centrifugation at $1,500 \mathrm{rpm}, 5 \mathrm{~min}$, and suspended in PBS at $25 \mathrm{mg} / \mathrm{ml}$. Since these preparations were occasionally toxic to the target cells, they were routinely treated with the protease inhibitor 6-amidino-2-naphthyl-4guanodinobenzoate dihydrochloride (27) at $25 \mu \mathrm{g} / \mathrm{ml}$ for $10 \mathrm{~min}$, at $25^{\circ} \mathrm{C}$. The opsonized zymosan was again washed twice in PBS and used the same day. PMA (Sigma) was dissolved at $10 \mu \mathrm{g} / \mathrm{ml}$ in dimethylsulfoxide and stored at $-20^{\circ} \mathrm{C}$. $\mathrm{O}_{2}^{-}$was determined by cytochrome $c$ reduction measured at $550 \mathrm{~nm}$ either $7-10 \mathrm{~min}$ after addition of activator or by using continuous kinetics measurements monitored in a spectrophotometer (Kontron, Zürich, Switzerland). Reactions were carried out in a final volume of $1.1 \mathrm{ml}$ at $37^{\circ} \mathrm{C}$ using reactant concentrations as shown in Results. Supplements to the assay mixture were added before addition of activator.

Myeloperoxidase activity was measured by a modification of the procedure of Bretz and Baggiolini (28). Neutrophils were suspended in Dulbecco's PBS at $6 \times 10^{6} / \mathrm{ml}$ containing $1 \mathrm{mg} / \mathrm{ml}$ glucose, $1 \mathrm{mg} / \mathrm{ml}$ human serum albumin. After treatment with activator for $15 \mathrm{~min}$ at $37^{\circ} \mathrm{C}, 20 \mu \mathrm{l}$ of serial twofold dilutions of the neutrophil free supernatant were added in replicates to wells of microtiter plates. The reaction was initiated by the addition of $200 \mu \mathrm{l}$ of $o$-dianisidine at $0.1 \mathrm{mg} / \mathrm{ml}$ in citrate buffer, $\mathrm{pH} 5.5,0.05 \%$ Triton X-100, $0.08 \mathrm{mM} \mathrm{H}_{2} \mathrm{O}_{2}$. A standard curve was generated using horseradish peroxidase (Sigma) at $0.005-0.02 \mathrm{U} / \mathrm{ml}$. The optical density at $450 \mathrm{~nm}$ was determined at 15-30 min using an MR600 ELISA reader (Dynatech Laboratories, Alexandria, VA), and equivalent units of peroxidase activity were determined from the linear region of the standard curve.

Beta glucuronidase activity was assayed by modification of the method of Brittinger et al. (29). Supernatant from activated neutro- phils was collected as described for the myeloperoxidase assay and 50 $\mu \mathrm{l}$ was added to wells of a microtiter plate. The reaction was initiation by addition of a substrate solution consisting of $2.5 \mathrm{mM}$ phenolphthalein glucuronic acid in $0.15 \mathrm{M}$ acetate buffer, $\mathrm{pH}$ 4.6. Incubation was carried out overnight at room temperature and the amount of phenolphthalein released was determined at $550 \mathrm{~nm}$ after addition of $0.2 \mathrm{M}$ glycine buffer, $0.2 \mathrm{M} \mathrm{NaCl}, \mathrm{pH}$ 10.4. A standard curve was generated using known amounts of phenolphthalein; beta glucuronidase activity was expressed as Fishman units (30) (micrograms phenolphthalein released/hour).

HPLC analysis of procainamide metabolites. Neutrophils were suspended in Dulbecco's PBS containing $7.5 \mathrm{mM}$ glucose and $1 \mathrm{mM}$ ascorbic acid in the presence of $30-50 \mu \mathrm{M}$ procainamide and 7-10 $\mu \mathrm{M}$ $\mathrm{N}$-acetyl procainamide (NAPA) in a final volume of $0.5 \mathrm{ml}$. The activator was added and the mixture incubated at $37^{\circ} \mathrm{C}, 30 \mathrm{~min}$ and forced through a $0.45-\mu \mathrm{m}$ fluoropolymer filter (Gelman Sciences, Ann Arbor, MI) into a microfuge tube prefrozen in dry ice. These samples froze within $<1 \mathrm{~min}$ and were stored at $-70^{\circ} \mathrm{C}$ until analysis.

HPLC analysis was performed with a Gilson modular system using a $\mathrm{C}^{18}$-reverse-phase column (Bio-Rad Laboratories, Richmond, CA; RP-304). In general, $150 \mu$ fresh or rapidly thawed sample was applied to the column equilibrated in $4 \%$ acetonitrile, $0.2 \%$ acetic acid, $0.01 \%$ triethylamine at $1.0 \mathrm{ml} / \mathrm{min}$. The mobile phase (solvent B) consisted of $80 \%$ acetonitrile, $4 \%$ acetic acid, $0.2 \%$ triethylamine in water. 9 min after sample injection a step gradient of $12.5 \%, 20 \%$ and finally $100 \%$ B was applied over the course of $27 \mathrm{~min}$. The flow rate was increased to $1.5 \mathrm{ml} / \mathrm{min}$ during the column wash period in $100 \% \mathrm{~B}$ before returning to normal loading condition after a total of $35 \mathrm{~min}$. The eluant was monitored at $270 \mathrm{~nm}$ at a sensitivity setting of 0.02 absorbance units full scale. Standards of procainamide and its analogues were obtained as previously described (13). Nitrosoprocainamide was a gift from Dr. Evelyn Hess, University of Cincinnati Medical Center. NAPA was used as an internal standard in every analysis to monitor recoveries. Peaks were quantified by area integration and converted to nanomoles of analogue based on similar analyses using known concentrations of the respective analogue. In some cases samples were pretreated with $0.1 \mathrm{~N} \mathrm{NaOH}$ for 5 min followed by neutralization with $0.1 \mathrm{~N}$ acetic acid to oxidize PAHA before analysis.

Cytotoxicity assay. Detection of cytotoxic metabolites of procainamide was based on previous studies in which cell lines exposed to procainamide analogues were assessed for viability by $\left[{ }^{3} \mathrm{H}\right]$ thymidine incorporation into DNA. Viability as measured by DNA synthesis correlated with trypan blue dye exclusion and tetrazolium dye reduction (13). The cytotoxicity assay was based on the capacity of activated neutrophils to kill GM4672B cells (Human Genetic Mutant Cell Repository Institute for Medical Research, Camden, NJ) when procainamide was present. In the standard assay, $0.5 \mathrm{ml}$ log phase, washed GM4672B cells in the presence or absence of procainamide (E. R. Squibb \& Sons, Princeton, NJ) were mixed with $0.2 \mathrm{ml}$ of various concentrations of neutrophils in $12 \times 75 \mathrm{~mm}$ polypropylene tubes followed by addition of $30 \mu \mathrm{l}$ of opsonized zymosan at $25 \mathrm{mg} / \mathrm{ml}$. Opsonized zymosan was sterilized by $3,200 \mathrm{R} \gamma$-irradiation. The final concentration of reactants was generally $1.5 \times 10^{5} \mathrm{GM} 4672 \mathrm{~B}$ cells $/ \mathrm{ml}$, $0.3-1.2 \times 10^{6}$ neutrophils $/ \mathrm{ml}, 50 \mu \mathrm{M}$ procainamide, $7.5 \mu \mathrm{Ci} / \mathrm{ml}\left[{ }^{3} \mathrm{H}\right]-$ thymidine (ICN Radiochemicals) and $1.0 \mathrm{mg} / \mathrm{ml}$ opsonized zymosan all in DME containing high glucose concentration and $10 \%$ fetal bovine serum (Hyclone Labs, Logan, UT). Tubes were loosely capped and placed in a $37^{\circ} \mathrm{C}$ incubator equilibrated with $10 \% \mathrm{CO}_{2}$ and harvested in $0.1-\mathrm{ml}$ quadruplicate aliquots the following day as previously described (13).

In some experiments a two-stage assay was performed in which $\left[{ }^{3} \mathrm{H}\right]$ thymidine was absent during a $1-\mathrm{h}$ first stage that contained all the other reactants followed by centrifugation and replacement of the medium with complete medium containing $\left[{ }^{3} \mathrm{H}\right]$ thymidine. The role of $\mathrm{NaCl}$ was examined in this two-stage assay by replacing the standard medium in the first stage with a chloride-free medium, consisting of $0.25 \mathrm{M}$ sucrose, $7.5 \mathrm{mM}$ glucose, $0.01 \mathrm{M}$ sodium phosphate, $0.5 \mathrm{mM}$ calcium lactate, $0.5 \mathrm{mM}$ magnesium sulfate and $10 \%$ fetal bovine 
serum exhaustively dialyzed against chloride-free medium using a 2,000 D cutoff membrane. Opsonized zymosan was washed in chloride-free medium before use.

Cell-free oxidation of procainamide. The capacity of myeloperoxidase (MPO) to mediate oxidation of procainamide was evaluated by HPLC. MPO was isolated from normal neutrophils disrupted by nitrogen cavitation as previously described (19). Briefly, azurophil granules were banded by discontinuous Percoll density gradient ultracentrifugation and washed free of Percoll by centrifugation in an SW41Ti rotor at $37,000 \mathrm{rpm}, 90 \mathrm{~min}, 5^{\circ} \mathrm{C}$. Granules were disrupted by sonication and stored in $0.34 \mathrm{M}$ sucrose at $-70^{\circ} \mathrm{C}$. MPO activity was determined exactly as performed on neutrophil supernatants. Procainamide oxidation experiments were initially optimized with respect to MPO and substrate concentrations and incubation duration. Reactions were generally performed in $12 \times 75 \mathrm{~mm}$ polypropylene tubes in a final volume of $0.5 \mathrm{ml}$. The standard conditions consisted of procainamide and NAPA at 30 and $7 \mu \mathrm{M}$, respectively, in Dulbecco's PBS followed by addition of MPO to a final concentration of $5 \mathrm{mU} / \mathrm{ml}$. Ascorbic acid to $1 \mathrm{mM}$ and hypoxanthine (HX) to $0.1 \mathrm{mM}$ were added in some experiments. The reaction was initiated by addition of either $\mathrm{H}_{2} \mathrm{O}_{2}$ or xanthine oxidase (Sigma, grade III). After $30 \mathrm{~min}$ at $37^{\circ} \mathrm{C}$, the reaction was terminated by ultrafiltration onto dry ice and analyzed by HPLC as previously described. In some experiments Dulbecco's PBS was replaced with $0.01 \mathrm{M}$ phosphate buffer, $\mathrm{pH} 7.4$ with or without $0.14 \mathrm{M}$ $\mathrm{NaCl}$.

\section{Results}

A human lymphoblastoid B-cell line, GM4672B, was used as the bioindicator for the presence of cytotoxic products by measurement of $24 \mathrm{~h}\left[{ }^{3} \mathrm{H}\right]$ thymidine incorporation into DNA. As shown in Fig. 1 procainamide caused a nonlinear dose-dependent decrease in indicator cell viability in the presence of 0.3 $\times 10^{6} / \mathrm{ml}$ normal human neutrophils activated with opsonized zymosan. NAPA was largely noncytotoxic in this system except at $0.33 \mathrm{mM}$, indicating that the free amino group on procainamide was required to generate cytotoxic products. In the absence of neutrophils or opsonized zymosan, $0.33 \mathrm{mM}$ procainamide was nontoxic. These results suggest that neutrophils can metabolize procainamide to a cytotoxic product well within the range of procainamide concentrations therapeutically employed $(0.03-0.05 \mathrm{mM})$.

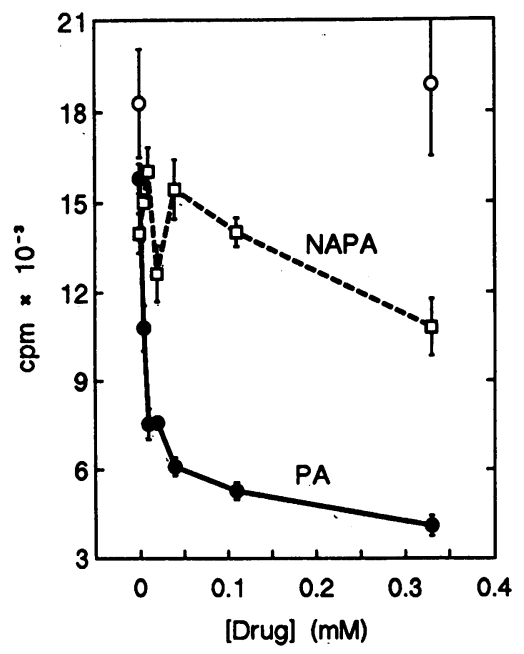

Figure 1. Production of cytotoxic procainamide by activated neutrophils. Mixtures of 1 $\times 10^{5} / \mathrm{ml} \mathrm{GM4672B}$ target cells, $3 \times 10^{5} / \mathrm{ml}$ neutrophils and increasing concentrations of procainamide (PA) or $\mathrm{N}$-acetyl procainamide (NAPA) were activated with $1 \mathrm{mg} / \mathrm{ml}$ opsonized zymosan. After overnight incubation at $37^{\circ} \mathrm{C}$ in the presence of $\left[{ }^{3} \mathrm{H}\right]$ thymidine, target cell viability was measured by scintillation counting. Viability in

the absence of neutrophils with or without opsonized zymosan is also shown (0). Error bars are $\pm 1 \mathrm{SD}$. Values are based on quadruplicate determinations in a single representative experiment.
We suspected that neutrophils oxidized procainamide to the hydroxylamine derivative (PAHA) because this analogue was previously shown to be cytotoxic at micromolar concentrations (13). Evidence for production of PAHA by neutrophils was obtained by HPLC analysis as shown in Fig. 2. A standard mixture of procainamide and four analogues was separated by $\mathrm{C}^{18}$ reverse-phase chromatography; PAHA eluted at $6.1 \mathrm{~min}$, separated by $2.5 \mathrm{~min}$ from procainamide as shown in Fig. $2 \mathrm{~A}$. High pH oxidizes PAHA to nitroprocainamide (16) which eluted at $19.8 \mathrm{~min}$, and Fig. $2 B$ demonstrates the quantitative loss of PAHA and increase in nitro-PA when the standard mixture was treated with $0.1 \mathrm{~N} \mathrm{NaOH}$ before HPLC analysis. HPLC analysis of the medium derived from $1.8 \times 10^{6}$ neutrophils/ml activated with opsonized zymosan in the presence of $0.05 \mathrm{mM}$ procainamide and $0.01 \mathrm{mM}$ NAPA is shown in Fig. $2 C$. In the presence of neutrophils a peak appeared that co-eluted with PAHA; treatment with $0.1 \mathrm{~N} \mathrm{NaOH}$ largely eliminated this peak and created a small amount of nitro-PA as shown in Fig. $2 \mathrm{D}$, consistent with the behavior of authentic PAHA (Fig. $2 B$ ). If procainamide, neutrophils or opsonized zymosan is omitted from the incubation mixture, no PAHA peak appears (data not shown). NAPA, which elutes at 15.2 min, was not a precursor for PAHA and served as an internal standard. These results indicate that the free amino group on procainamide was metabolized to a hydroxylamine by activated, but not by unstimulated neutrophils.

Inhibition of cytotoxicity was commonly observed when the neutrophil concentration exceeded $1 \times 10^{6} / \mathrm{ml}$. Fig. 3 shows typical results of a donor displaying maximum cytotox-

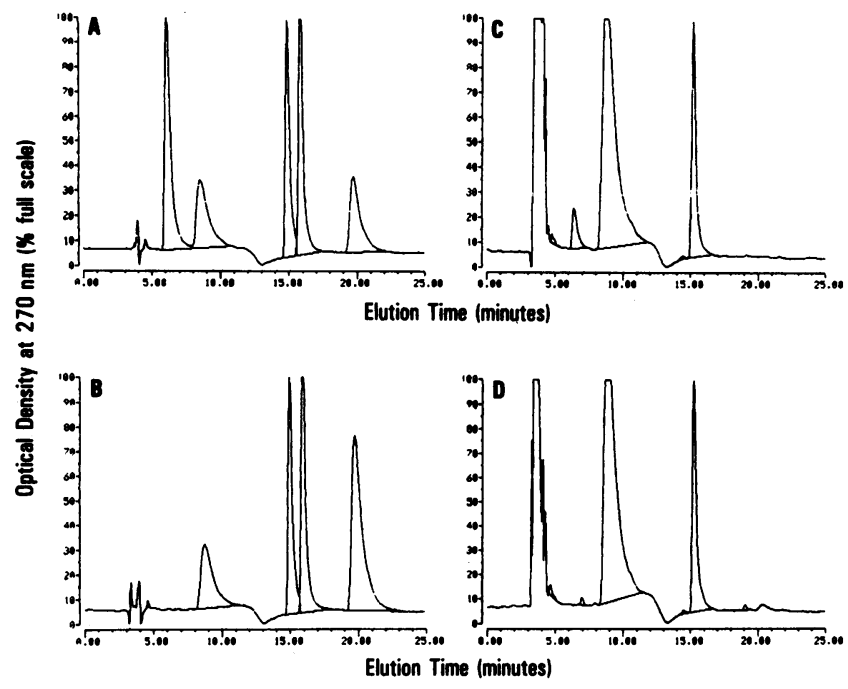

Figure 2. Generation of PAHA by activated neutrophils. HPLC of a standard mixture of procainamide analogues ( $33 \mu \mathrm{M}$ each, $50 \mu \mathrm{l}$ injection volume) is shown in the left panels before $(A)$ and after $(B)$ treatment with $0.1 \mathrm{M} \mathrm{NaOH}$ followed by neutralization. Full scale $=0.02$ O.D. units. Peaks corresponded exactly to the elution positions of the analogues chromatographed separately. Starting from PAHA at $6.1 \mathrm{~min}$, the next four peaks are procainamide, NAPA, NAPA- $N$-oxide, and nitro-procainamide. High pH converts PAHA to nitro-procainamide. $C$ and D are chromatographs of $150 \mu \mathrm{l}$ of supernatant from neutrophils activated with opsonized zymosan at 1.8 $\times 10^{6}$ cells $/ \mathrm{ml}$ in the presence of $50 \mu \mathrm{M}$ procainamide, $10 \mu \mathrm{M}$ NAPA and $1 \mathrm{mM}$ ascorbic acid. D shows supernatant pretreated with $0.1 \mathrm{M} \mathrm{NaOH}$ before HPLC analysis. The large peak in C and D at 3-4 $\mathrm{min}$ is ascorbic acid, which elutes in the void volume. 


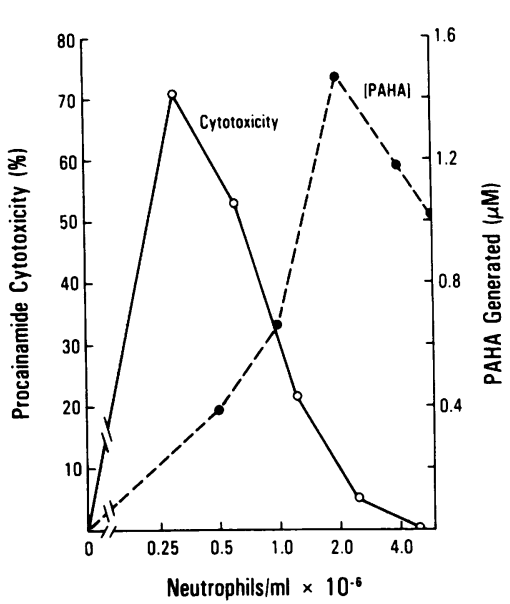

Figure 3. Stoichiometry of procainamide cytotoxicity and PAHA production. The standard cytotoxicity assay was performed in increasing concentrations of neutrophils activated with opsonized zymosan. PAHA generation was quantified by HPLC analysis on supernatant from increasing concentrations of neutrophils activated with opsonized zymosan in the presence of $0.03 \mathrm{mM}$ procainamide.

icity response at $0.3 \times 10^{6}$ neutrophils $/ \mathrm{ml}$ followed by a stoichiometric decrease with increasing neutrophil concentration, reaching negligible cytotoxicity at $4.8 \times 10^{6}$ neutrophils $/ \mathrm{ml}$. As also shown in this figure, suppression of cytotoxicity was not due to decrease in the amount of PAHA generated since PAHA production showed a linear increase with neutrophil concentration up to $2.4 \times 10^{6} / \mathrm{ml}$ (slope of linear regression plot $=0.62, r=0.99$ by least square analysis). Similar results were obtained when activation with opsonized zymosan was replaced by PMA except that two-thirds less cytotoxicity and $50 \%$ less PAHA production were observed (slope $=0.23 \mu \mathrm{M}$ [PAHA]/[neutrophil] $\times 10^{-6}, r=0.98$ ) (data not shown).

The curious inhibition of procainamide cytotoxicity when neutrophil concentration exceeded $\sim 1 \times 10^{6} / \mathrm{ml}$ was consistently observed with most donors although the exact cell density for maximum cytotoxicity varied from donor to donor. In contrast to neutrophil mediated procainamide cytotoxicity, PAHA production increased with increasing neutrophil concentration as shown in Fig. 3. This suggested the possibility that a factor was released from neutrophils which protected target cells from the toxic action of PAHA. Such a phenomenon was supported by the experiment shown in Table I. When supernatant from increasing concentrations of activated neutrophils was added to viable neutrophils, procainamide cytotoxicity was concomitantly decreased. Inhibition of neutrophil mediated procainamide cytotoxicity was not paralleled by a decrease in PAHA accumulation which remained unaffected by addition of neutrophil supernatant (Table I). These results demonstrate the presence of an inhibitor of the cytotoxic procainamide metabolites, released in a dose-dependent fashion from activated neutrophils.

Role of neutrophil oxidative metabolism. The major events associated with neutrophil activation are a respiratory burst and lysosomal degranulation resulting in production of superoxide anion and release of myeloperoxidase into the extracellular environment. The role of these mediators in procainamide oxidation and cytotoxicity was explored by the use of competing enzymes and inhibitors of these processes. As shown in Table II superoxide dismutase (SOD) quantitatively eliminated $\mathrm{O}_{2}^{-}$generated after activation of neutrophils with opsonized zymosan without inhibiting release of the azurophil granule enzymes MPO and beta glucuronidase. Hydrogen peroxide $\left(\mathrm{H}_{2} \mathrm{O}_{2}\right)$, the product of SOD action on $\mathrm{O}_{2}^{-}$, can be hydrolyzed by addition of catalase; at a catalase concentration of

Table I. Inhibition of Neutrophil-mediated Procainamide Cytotoxicity by Supernatant from Activated Neutrophils

\begin{tabular}{cccc}
\hline & \multicolumn{2}{c}{$\begin{array}{c}\text { Procainamide } \\
\text { cytotoxicity }\end{array}$} & \\
\cline { 2 - 3 } $\begin{array}{c}\text { [Neutrophil] of } \\
\text { supernatant source }\end{array}$ & Exp. 1 & Exp. 2 & PAHA generated \\
\hline & & & $\mu M$ \\
0 & $46 \%$ & $63 \%$ & 0.8 \\
$0.6 \times 10^{6} / \mathrm{ml}$ & $46 \%$ & $61 \%$ & ND \\
$1.2 \times 10^{6} / \mathrm{ml}$ & $28 \%$ & $46 \%$ & 0.8 \\
$2.4 \times 10^{6} / \mathrm{ml}$ & $15 \%$ & $41 \%$ & 0.8 \\
$4.8 \times 10^{6} / \mathrm{ml}$ & $30 \%$ & $29 \%$ & 0.9 \\
\hline
\end{tabular}

Supernatant from increasing concentrations of activated neutrophils was added to viable neutrophils at $0.6 \times 10^{6} / \mathrm{ml}$ in Exp. 1 and 0.4 $\times 10^{6} / \mathrm{ml}$ in Exp. 2 in the presence or absence of $0.03 \mathrm{mM}$ procainamide in the standard cytotoxicity assay. The donor from Exp. 1 was also used to measure PAHA production by HPLC in the presence of $0.03 \mathrm{mM}$ procainamide. Concentrations refer to the final reaction mixture.

$200 \mathrm{U} / \mathrm{ml}$, MPO activity was still detectable when an exogenous source of $\mathrm{H}_{2} \mathrm{O}_{2}$ was supplied (Table II), but no longer detectable at $2,000 \mathrm{U} / \mathrm{ml}$ catalase (data not shown). MPO was also specifically inhibited by $0.2 \mathrm{mM} \mathrm{NaN}_{3}$ without affecting $\mathrm{O}_{2}^{-}$generation or beta glucuronidase activity.

Table III shows the effects of these inhibitors and enzymes on the production of PAHA and the generation of cytotoxic procainamide. Consistent with previous results, detection of PAHA and procainamide cytotoxicity was completely dependent on neutrophil activation, and opsonized zymosan supported the latter process considerably better than did PMA. SOD caused a slight increase in both PAHA generation and cytotoxicity indicating that $\mathrm{O}_{2}^{-}$was not a direct reactant. The pivotal role of $\mathrm{H}_{2} \mathrm{O}_{2}$ and MPO was demonstrated by the almost total inhibition of both PAHA generation and procain-

Table II. Effect of Various Inhibitors on Oxidative Metabolism and on Degranulation Reactions of Activated Neutrophils

\begin{tabular}{lccc}
\hline \multicolumn{1}{c}{ Additions } & $\mathrm{O}_{2}^{-}$ & MPO & $\beta$-glucuronidase \\
& $n$ mol/7 min & $\mathrm{mU} / \mathrm{ml}$ & $U / \mathrm{ml}$ \\
None & 0 & 3 & 0.04 \\
Op. zy. & 9.7 & 19 & 0.4 \\
Op. zy. + NaN ${ }_{3}$ & 10.3 & 0 & 0.3 \\
Op. zy. + SOD & 0.1 & 12 & 0.3 \\
Op. zy. + catalase & 10.7 & 14 & 0.5 \\
Op. zy. + d. catalase & 9.6 & 18 & 0.4 \\
PMA & 51.5 & 5 & 0.1 \\
& & & \\
\hline
\end{tabular}

Neutrophils were activated in Dulbecco's PBS, $7.5 \mathrm{mM}$ glucose, 1 $\mathrm{mg} / \mathrm{ml}$ albumin plus the various additives at $3 \times 10^{6}$ neutrophils $/ \mathrm{ml}$ for $\mathrm{O}_{2}^{-}$and $6 \times 10^{6}$ neutrophils $/ \mathrm{ml}$ for MPO and beta glucuronidase assays. Typical results from a single donor. The final concentration of each additive was: $1 \mathrm{mg} / \mathrm{ml}$ opsonized zymosan (op. zy.) when [neutrophil] $<=3 \times 10^{6} / \mathrm{ml}$ and $2 \mathrm{mg} / \mathrm{ml}$ at $6 \times 10^{6}$ neutrophils $/ \mathrm{ml}$; $\left[\mathrm{NaN}_{3}\right]=0.2 \mathrm{mM}$; [SOD] $=20 \mu \mathrm{g} / \mathrm{ml}$; [catalase] $=200 \mathrm{U} / \mathrm{ml} ;$ PMA $=50 \mathrm{ng} / \mathrm{ml}$. Heat denatured catalase is indicated by " $\mathrm{d}$." 
Table III. Effect of Activators and Inhibitors of Oxidative Metabolism on Neutrophil-mediated PAHA Generation and Procainamide Cytotoxicity

\begin{tabular}{lcccc}
\hline \multicolumn{1}{c}{ Additions } & \multicolumn{2}{c}{ PAHA generated } & \multicolumn{2}{c}{$\begin{array}{c}\text { Procainamide } \\
\text { cytotoxicity }\end{array}$} \\
& $n$ & $\mu M \pm S E M$ & $n$ & $\% \pm S D$ \\
None & 4 & $0.04 \pm 0.04$ & 4 & $1 \pm 2$ \\
Op. zy. & 6 & $1.52 \pm 0.23$ & 3 & $63 \pm 13$ \\
Op. zy. + NaN & 3 & $0.14 \pm 0.04$ & 1 & $(8)$ \\
Op. zy. + SOD & 3 & $1.92 \pm 0.22$ & 3 & $73 \pm 6$ \\
Op. zy. + catalase & 4 & $1.18 \pm 0.27$ & 3 & $11 \pm 3$ \\
Op. zy. + d. catalase & & ND & 3 & $68 \pm 8$ \\
PMA & 2 & $1.27 \pm 0.51$ & 4 & $22 \pm 6$ \\
& & & & \\
\hline
\end{tabular}

PAHA production was measured under conditions similar to those used in Table Il with the addition of $1 \mathrm{mM}$ ascorbic acid and 0.05 $\mathrm{mM}$ procainamide using $2 \times 10^{6}$ neutrophils $/ \mathrm{ml}$. The standard cytotoxicity assay (i.e., with and without $0.03 \mathrm{mM}$ procainamide) was supplemented with sterile forms of the additives, and the average percent cytotoxicity at $0.3 \times 10^{6}$ neutrophils $/ \mathrm{ml}$ is tabulated. $n$, number of experiments using different donors. ND, not done.

amide cytotoxicity by $\mathrm{NaN}_{3}$. However, neutrophils in the absence of procainamide were $85 \%$ toxic to the target cells if $\mathrm{NaN}_{3}$ was present for $1 \mathrm{~h}$ after initiation of activation presumably due to accumulation of $\mathrm{H}_{2} \mathrm{O}_{2}$, but the simultaneous presence of procainamide produced only $8 \%$ additional toxicity under these conditions. In general a good agreement between inhibition of PAHA generation and inhibition of procainamide cytotoxicity was observed. The only disparity was the effect of $200 \mathrm{U}$ catalase/ml, which largely blocked procainamide cytotoxicity but decreased PAHA generation by only $22 \%$, an insignificant difference. This effect of catalase on procainamide cytotoxicity was due to its enzymatic activity since heat denatured catalase did not block procainamide cytotoxicity. At all neutrophil concentrations up to $4.8 \times 10^{6} / \mathrm{ml}$ catalase completely quenched neutrophil-mediated procainamide cytotoxicity (data not shown). These results suggest that MPO released during degranulation mediates the oxidation of procainamide to cytotoxic PAHA.

Hydrogen peroxide, the primary substrate of MPO, is derived from the dismutation of $\mathrm{O}_{2}^{-}$generated during the respiratory burst. Since neutrophils from patients with chronic granulomatous disease (CGD) lack or have a greatly diminished capacity to produce $\mathrm{O}_{2}^{-}$and therefore $\mathrm{H}_{2} \mathrm{O}_{2}$, the role of these potential reactants in procainamide oxidation can be determined. Two patients were studied. CGD1 was a male with the $\mathrm{X}$-linked/cytochrome $\mathrm{b}_{558}$ negative form of CGD and had no capacity to generate $\mathrm{O}_{2}^{-}$as shown in Table IV; CGD2 was a female with autosomal recessive/cytochrome $b_{558}$ negative disease and produced a small amount of $\mathrm{O}_{2}^{-}(1-2 \%$ of normal). The capacity of neutrophils from each of these patients to generate PAHA was greatly reduced when compared to normal controls tested on the same day (Table IV). Neutrophils from both patients failed to mediate cytotoxic procainamide formation as shown in Fig. 4. These results indicate that procainamide oxidation requires $\mathrm{O}_{2}^{-}$released during the respiratory burst. However, since SOD did not inhibit procainamide cytotoxicity or PAHA generation by normal neutrophils, $\mathrm{O}_{2}^{-}$ serves only as a precursor for $\mathrm{H}_{2} \mathrm{O}_{2}$.
Table IV. CGD vs. Normal Neutrophils: Correlation between $\mathrm{O}_{2}^{-}$ and PAHA Production

\begin{tabular}{|c|c|c|c|}
\hline Exp. No. & Neutrophils & $\mathrm{O}_{2}^{-}$production* & PAHA generated ${ }^{\ddagger}$ \\
\hline & & $\mathrm{nmol} / \mathrm{min} / 3 \times 10^{6} \mathrm{cells}$ & $\mu M$ \\
\hline \multirow[t]{2}{*}{1} & Normal & 9.8 & 2.20 \\
\hline & CGD1 & 0.0 & 0.16 \\
\hline \multirow[t]{2}{*}{2} & Normal & 17.6 & 0.90 \\
\hline & CGD2 & 0.2 & 0.05 \\
\hline
\end{tabular}

* Activator = PMA.

${ }^{\ddagger}$ Neutrophils activated at $2 \times 10^{6} / \mathrm{ml}$ with opsonized zymosan.

Procainamide oxidation in a cell free system. The previous results suggested that isolated MPO should be able to mediate the oxidation of procainamide to PAHA in the presence of $\mathrm{H}_{2} \mathrm{O}_{2}$. This was confirmed using either direct addition of $\mathrm{H}_{2} \mathrm{O}_{2}$ or an $\mathrm{H}_{2} \mathrm{O}_{2}$ generating system, xanthine oxidase (XO) plus hypoxanthine (HX), as shown in Table V. Concentrations of $\leq 0.1 \mathrm{mM} \mathrm{H}_{2} \mathrm{O}_{2}$ in the absence of MPO did not oxidize procainamide (data not shown). PAHA was detected after incubation of procainamide with MPO and $0.03 \mathrm{mM} \mathrm{H}_{2} \mathrm{O}_{2}$ if ascorbic acid was added to a final concentration of $1 \mathrm{mM}$; in the absence of ascorbic acid no PAHA was detected but larger amounts of nitro-PA and a peak eluting at $5 \mathrm{~min}$ appeared. This peak coelutes with the nitroso derivative of procainamide, which is derived from the spontaneous oxidation of PAHA in the absence of ascorbic acid (16). Similar results were obtained with $\mathrm{HX}$ and $5 \mathrm{mU} / \mathrm{ml} \mathrm{XO}$. This XO concentration generated $1.2 \mathrm{nmol} \mathrm{O}-/ \mathrm{min}$ in the cytochrome $c$ reduction assay, similar to the $\mathrm{O}_{2}^{-}$producing capacity of $6 \times 10^{6}$ neutrophils/ml activated with opsonized zymosan (Table II). Appearance of PAHA, nitro-PA or presumed nitroso-PA was prevented by omission of procainamide or MPO or by the use of boiled MPO or XO (data not shown). Although we could not test the cytotoxic properties of these mixtures due to toxicity of the reactants in the absence of procainamide, the HPLC analyses of this cell free system are compatible with results obtained using intact neutrophils demonstrating the key role of MPO in generation of cytotoxic procainamide.

Hypochlorite ion is not responsible for procainamide oxidation to the hydroxylamine. In the presence of chloride ions the primary product of MPO-mediated $\mathrm{H}_{2} \mathrm{O}_{2}$ hydrolysis is

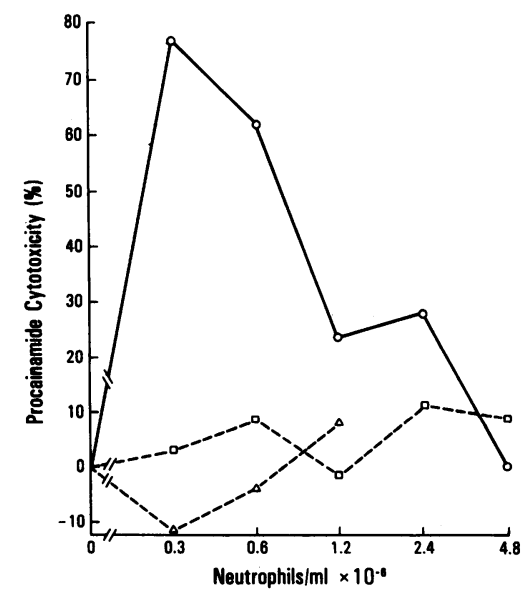

Figure 4. Inability of neutrophils from patients with chronic granulomatous disease to support procainamide cytotoxicity. The standard cytotoxicity assay was performed using normal neutrophils (o), neutrophils from a patient with $X$ linked CGD (), or neutrophils from a patient with autosomal recessive CGD $(\Delta)$. 
Table V. MPO-mediated, Cell Free Procainamide Oxidation

\begin{tabular}{|c|c|c|c|c|c|}
\hline \multirow[b]{2}{*}{ Primary substrate } & \multirow{2}{*}{$\begin{array}{c}\text { Ascorbic } \\
\text { acid }\end{array}$} & \multirow[b]{2}{*}{$n$} & \multicolumn{3}{|c|}{ Products detected $(\mu \mathrm{M} \pm \mathrm{SD})$} \\
\hline & & & PAHA & Nitro-Pa & (Nitroso-PA) \\
\hline $0.03 \mathrm{mM} \mathrm{H}_{2} \mathrm{O}_{2}$ & - & 3 & 0.00 & $1.21 \pm 0.14$ & $4.95 \pm 0.98$ \\
\hline $\begin{array}{l}0.03 \mathrm{mM} \mathrm{H}_{2} \mathrm{O}_{2} \\
0.10 \mathrm{mM} \mathrm{HX}\end{array}$ & + & 3 & $0.75 \pm 0.09$ & $0.03 \pm 0.05$ & 0.00 \\
\hline$+5 \mathrm{mU} / \mathrm{ml} \mathrm{XO}$ & - & 3 & 0.00 & 0.00 & $0.93 \pm 0.13$ \\
\hline $0.10 \mathrm{mM} \mathrm{HX}$ & & & & & \\
\hline$+5 \mathrm{mU} / \mathrm{ml} \mathrm{XO}$ & + & 4 & $0.79 \pm 0.13$ & 0.00 & 0.00 \\
\hline
\end{tabular}

In addition to the primary substrate and $1 \mathrm{mM}$ ascorbic acid as shown, all reaction mixtures contained $25 \mathrm{mU} / \mathrm{ml} \mathrm{MPO}, 0.03 \mathrm{mM}$ PA and $0.007 \mathrm{mM}$ NAPA in Dulbecco's PBS. $\mathrm{H}_{2} \mathrm{O}_{2}$ or XO was added last. Mixtures were incubated at $37^{\circ} \mathrm{C}$ for $30 \mathrm{~min}$ with $\mathrm{XO}$ or for 60 min with $\mathrm{H}_{2} \mathrm{O}_{2}$ before ultrafiltration and HPLC analysis. $n$, number of separate experiments.

$\mathrm{OCl}^{-}(31)$, a strong oxidizing agent which could be responsible for procainamide oxidation by activated neutrophils. Although $0.1 \mathrm{mM}$ procainamide treated with $0.2 \mathrm{mM} \mathrm{NaOCl}$ was $15 \%$ cytotoxic to GM4672B cells, no PAHA was detected by HPLC analysis, but two peaks eluting at 25 and 30 mins appeared, possibly corresponding to mono- and dichloramine procainamide. Procainamide was completely oxidized by 0.35 $\mathrm{mM} \mathrm{NaOCl}$, but this $\mathrm{NaOCl}$ concentration was highly toxic to the target cells by itself. At $\leq 0.1 \mathrm{mM} \mathrm{NaOCl}$, concentrations more likely to develop in the extracellular medium of activated neutrophils in the bioassay, no procainamide oxidation was observed.

To directly test whether $\mathrm{OCl}^{-}$may be involved in neutrophil-mediated oxidation of procainamide, we measured the generation of cytotoxic PAHA in the presence or absence of $0.14 \mathrm{M} \mathrm{NaCl}$. Since the bioassay requires overnight incubation of the target cells in complete tissue culture medium, a two stage assay was performed in which the mixture of neutrophils and target cells in either complete medium or in chloride-free isotonic medium (see Methods) was resuspended in complete medium containing $\left[{ }^{3} \mathrm{H}\right]$ thymidine $1 \mathrm{~h}$ after activation with opsonized zymosan. In complete medium in the 2 stage assay, target cell killing due to the presence of procainamide in the activation step was $57 \%, 58 \%$, and $37 \%$ at $0.3,0.6$, and 1.2 $\times 10^{6}$ neutrophil $/ \mathrm{ml}$, respectively, compared to $57 \%, 43 \%$, and $15 \%$ in the standard one stage assay performed in parallel. When the first stage was done in chloride-free medium, activated neutrophils were $>50 \%$ cytotoxic in the absence of procainamide, so the capacity of neutrophils to metabolize procainamide to a cytotoxic product could not be evaluated. However, by HPLC analysis $1.1 \mu \mathrm{M}$ PAHA was detected in the chloride-free medium compared to $1.3 \mu \mathrm{M}$ PAHA in the standard chloride-containing medium, suggesting that $\mathrm{OCl}^{-}$was not involved in oxidation of procainamide to PAHA by neutrophils. $\left(\mathrm{O}_{2}^{-}\right.$production in response to opsonized zymosan in chloride-free medium was $40 \%$ below normal.) However, since intracellular chloride cannot be removed, this experiment does not absolutely eliminate a role of $\mathrm{OCl}^{-}$in procainamide oxidation.

The capacity of MPO in the cell-free system to support procainamide oxidation to PAHA facilitated examination of the role of $\mathrm{OCl}^{-}$in this process. HPLC analysis of procainamide treated with MPO in the presence or absence of $\mathrm{NaCl}$ using xanthine oxidase as the $\mathrm{H}_{2} \mathrm{O}_{2}$ generating system is shown in Table VI. It is clear from these data that $\mathrm{NaCl}$ was not required for PAHA generation. In fact, omission of $\mathrm{NaCl}$ resulted in a greater than two times enhancement in MPO-generated PAHA, perhaps due to $\mathrm{OCl}^{-}$-mediated destruction of PAHA. PAHA accumulation increased stoichiometrically with MPO concentration, indicating that $\mathrm{MPO}$ at $25 \mathrm{mU} / \mathrm{ml}$ was rate limiting in this reaction. These results support the findings with neutrophil mediated procainamide oxidation in the absence of $\mathrm{NaCl}$ as well as the inactivity of physiological concentrations of reagent $\mathrm{OCl}^{-}$, indicating that $\mathrm{OCl}^{-}$is not involved in neutrophil-mediated procainamide oxidation to PAHA.

\section{Discussion}

A proposed scheme for procainamide metabolism by neutrophils is shown in Fig. 5. Activation of neutrophils with opsonized zymosan causes release of $\mathrm{O}_{2}^{-}$into the extracellular environment, and $\mathrm{O}_{2}^{-}$dismutates into $\mathrm{H}_{2} \mathrm{O}_{2}$ either spontaneously or catalytically (via SOD). $\mathrm{H}_{2} \mathrm{O}_{2}$ is the primary substrate for MPO, which is exocytosed from azurophil granules during phagocytosis of opsonized zymosan. It is proposed that procainamide serves as a secondary substrate, reducing the heme group(s) in MPO back to ground state by donating two electrons and becoming oxidized to PAHA in the process.

Evidence for the linkage of procainamide oxidation with MPO action on $\mathrm{H}_{2} \mathrm{O}_{2}$ includes the observation that $\mathrm{NaN}_{3}$, a potent inhibitor of heme proteins such as MPO (31), blocks cytotoxicity and PAHA production, and that the copresence of the $\mathrm{H}_{2} \mathrm{O}_{2}$ hydrolyzing enzyme catalase eliminates the capacity of activated neutrophils to metabolize procainamide to a cytotoxic product. These conclusions are also supported by the cell free system in which activated neutrophils were replaced by the combination of MPO and either $\mathrm{H}_{2} \mathrm{O}_{2}$ or an $\mathrm{H}_{2} \mathrm{O}_{2}$-generating enzyme (XO). Procainamide oxidation to PAHA in the absence of neutrophils occurred at MPO and $\mathrm{O}_{2}^{-}$-generating activities similar to those observed when neutrophils were activated with opsonized zymosan. In the cell-free studies $\mathrm{H}_{2} \mathrm{O}_{2}$ was derived from $\mathrm{O}_{2}^{-}$, a product of xanthine oxidase action on hypoxanthine in contrast to activated neutrophils in which

Table VI. Effect of $\mathrm{NaCl}$ in MPO-mediated Procainamide Oxidation

\begin{tabular}{lccc}
\hline \multicolumn{1}{c}{ Medium } & [MPO] & $n$ & PAHA generated \\
\hline & $m U / m l$ & & $\mu M \pm S D$ \\
$0.14 \mathrm{M} \mathrm{NaCl}$ & 25 & 4 & $0.56 \pm 0.12$ \\
$0 \mathrm{NaCl}$ & 25 & 4 & $1.14 \pm 0.10$ \\
$0.14 \mathrm{M} \mathrm{NaCl}$ & 100 & 3 & $1.81 \pm 0.10$ \\
$0 \mathrm{NaCl}$ & 100 & 3 & $4.29 \pm 0.83$
\end{tabular}

Reaction conditions were similar to those in Table V except PBS was replaced with $0.01 \mathrm{M}$ phosphate buffer along with the $[\mathrm{NaCl}]$ shown. The source of $\mathrm{H}_{2} \mathrm{O}_{2}$ was $5 \mathrm{mU} \mathrm{XO} / \mathrm{ml}$ with $0.1 \mathrm{mM} \mathrm{HX}$, and $1 \mathrm{mM}$ ascorbic acid was present throughout. In the absence of either MPO or XO, no PAHA was generated. $n$, number of experiments performed on separate days. 


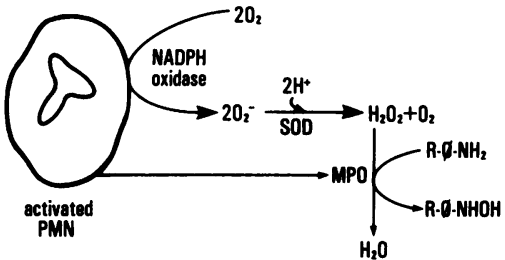

Figure 5. Proposed scheme for oxidation of procainamide to the hydroxylamine by activated neutrophils. $\mathrm{H}_{2} \mathrm{O}_{2}$ produced by the dismutation of $\mathrm{O}_{2}^{-}$serves as the primary substrate for MPO mediated cooxidation of the procainamide $\left(\mathrm{R}-\mathrm{NH}_{2}\right)$ to the hydroxylamine (R-NHOH).

$\mathrm{H}_{2} \mathrm{O}_{2}$ is derived from $\mathrm{O}_{2}^{-}$released during the respiratory burst. The necessity for a functioning respiratory burst in neutrophil-mediated metabolism of procainamide is based on the observation that neutrophils from patients with CGD with little or no capacity to produce $\mathrm{O}_{2}^{-}$were incapable of generating PAHA by biochemical and cytotoxicity criteria. Finally, the inability of physiological concentrations of $\mathrm{NaOCl}$ and the full capacity of chloride-free media to oxidize procainamide indicate that $\mathrm{OCl}^{-}$is not involved in procainamide oxidation to PAHA by neutrophils. Thus a number of independent lines of investigation provide consistent support for the scheme shown in Fig. 5.

In general there was a good agreement between procainamide-related cytotoxicity and PAHA generation under the optimum conditions for each system. However, substantial quantitative discrepancies between these assays were observed under some conditions such as low catalase concentration, during PMA-mediated activation and at high neutrophil concentration. In these cases $>1 \mu \mathrm{M}$ PAHA was detected but procainamide cytotoxicity was $22 \%$ with PMA, $11 \%$ with catalase and $<5 \%$ at $>2 \times 10^{6}$ neutrophils $/ \mathrm{ml}$. These discrepancies appear unrelated to the shorter incubation period for the HPLC studies (30 min) compared to the overnight incubation for the cytotoxicity assay, because generation of cytotoxic procainamide metabolites was complete by $1 \mathrm{~h}$ exposure of target cells to procainamide in the presence of activated neutrophils. However, previous studies demonstrated that PAHA was not directly cytotoxic but required further oxidation to the nitroso derivative, and ascorbic acid (which was present in the HPLC analyses of neutrophil metabolites) prevents such an oxidative reaction (13) (Table V). In the absence of ascorbic acid putative nitroso-PA was detected in the cell-free but not in the neutrophil system, perhaps due to formation of covalent bonds with components of the neutrophils themselves. This would suggest that the discordance between procainamide cytotoxicity and PAHA generation may be due to inefficient oxidation of PAHA to nitroso-PA.

Neutrophils contain $0.5 \mathrm{mM}$ ascorbic acid (32) which could be a candidate for the putative factor protecting target cells at high neutrophil concentrations, by blocking oxidation of PAHA to nitroso-PA. However, dilution effects and incomplete neutrophil cytolysis would seem to argue against such a role for neutrophil ascorbic acid, although it may be a contributing factor. A more likely candidate for the protective agent is $\mathrm{H}_{2} \mathrm{O}_{2}$, high concentrations of which may oxidize PAHA to noncytotoxic products such as nitro-PA or azoxy-procainamide (16). This would explain the discordance between PAHA generation and marginal cytotoxicity when neutrophils were activated with PMA or with opsonized zymosan at high neutrophil concentrations, since both conditions are associated with high $\mathrm{O}_{2}^{-}$and, therefore, $\mathrm{H}_{2} \mathrm{O}_{2}$ production. The nonenzymatic oxidation of PAHA by $\mathrm{H}_{2} \mathrm{O}_{2}$ is supported by the data in Table $\mathrm{V}$, demonstrating nitro- and more than five times as much nitroso-PA formation when an initially high $\mathrm{H}_{2} \mathrm{O}_{2}$ concentration was present $(30 \mu \mathrm{M})$, compared to an enzymatically generated $\mathrm{H}_{2} \mathrm{O}_{2}$ source. Thus, a multilevel role for $\mathrm{H}_{2} \mathrm{O}_{2}$ during MPO-mediated cooxidation of procainamide is proposed, in serving as the primary substrate for MPO, facilitating oxidation of PAHA to cytotoxic nitroso-PA, and at high concentrations inactivating PAHA by transformation to higher state oxidation products. Further studies will be needed to test these concepts.

The present studies establish the capacity of neutrophils to metabolize procainamide to reactive forms. Cytotoxicity of these metabolites per se may be important in the leukopenia occasionally observed in procainamide-induced lupus (33) and/or in release of nuclear debris from killed cells, which may then become immunogenic. However, subtoxic concentrations of procainamide metabolites may also be capable of altering lymphocyte function, as suggested by recent studies demonstrating hyperactivation of mitogen-treated lymphocytes exposed to sublethal concentrations of PAHA or nitroso-PA (34). It is also possible that PAHA may effect catabolism or processing of autoantigens, thereby increasing their localized concentration or possibly creating abnormal forms capable of initiating autoantibody production. Relevant to this possibility is the observation that patients with procainamideinduced lupus invariably develop IgG antibodies to a subnucleosome derivative of chromatin, the $\mathrm{H} 2 \mathrm{~A}-\mathrm{H} 2 \mathrm{~B}$ complex (35). The present demonstration of neutrophil-mediated metabolism of procainamide provides the precedent for a similar process by other phagocytic cells such as macrophage or by monocytes. Lymphoid compartments containing these cells may generate reactive forms of procainamide which may interfere with control of self-tolerance, leading to development of autoimmunity.

\section{Acknowledgments}

We thank Joyce Jones and Judy Zubar for expert technical assistance and Caryl Kane and the BCR Word Processing staff for assistance in the preparation of the manuscript. The gift of nitroso-procainamide from Dr. Evelyn V. Hess and her colleagues at the University of Cincinnati Medical Center is appreciated. We would also like to acknowledge the Journal reviewers' suggestions, especially the idea of measuring procainamide oxidation in chloride-free medium.

This work was supported in part by grant AR-34358 from the National Institutes of Health and U.S. Public Health Service grant RR-00833.

Note added in proof. Our more recent studies have indicated that the product identified in Table $V$ as nitro-PA actually elutes 1 min later at $21 \mathrm{~min}$. This corresponds to the elution time of nitroso-PA, which was previously misidentified as the 5 -min peak. The product eluting at 5 min has not been identified but may be PAHA- $N$-oxide. We thank Dr. Hess for helping to clarify this matter.

\section{References}

1. Lee, S. L., and P. H. Chase. 1975. Drug-induced systemic lupus erythematosus. A critical review. Semin. Arthritis Rheum. 5:83-103.

2. Weinstein, A. 1980. Drug-induced lupus erythematosus. Prog. Clin. Immunol. 4:1-21.

3. Woosley, R. L., D. E. Drayer, M. M. Reidenberg, A. S. Nies, K. Carr, and J. A. Oates. 1978. Effect of acetylator phenotype on the rate 
at which procainamide induces antinuclear antibodies and the lupus syndrome. N. Engl. J. Med. 298:1157-1159.

4. Lahita, R., J. Kluger, D. E. Drayer, D. Koffler, and M. M. Reidenberg. 1979. Antibodies to nuclear antigens in patients treated with procainamide or acetylprocainamide. N. Engl. J. Med. 301:1382-1385.

5. Roden, D. M., S. B. Reele, S. B. Higgins, G. R. Wilkinson, R. F. Smith, J. A. Oates, and R. L. Woosley. 1980. Antiarrhythmic efficacy, pharmacokinetics and safety of $\mathrm{N}$-acetylprocainamide in human subjects: comparison with procainamide. Am. J. Cardiol. 46:463-468.

6. Bluestein, H. G., N. J. Zvaifler, M. H. Weisman, and R. F. Shapiro. 1979. Lymphocyte alteration by procainamide: relation to drug-induced lupus erythematosus syndrome. Lancet. ii:816-819.

7. Miller, K. B., and D. Salem. 1982. Immune regulatory abnormalities produced by procainamide. Am. J. Med. 73:487-492.

8. Ochi, T., E. A. Goldings, P. E. Lipsky, and M. Ziff. 1983. Immunomodulatory effect of procainamide in man. J. Clin. Invest. 71:36-45.

9. De Boccardo, G., D. Drayer, A. L. Rubin, A. Novogrodsky, M. M. Reidenberg, and K. H. Stenzel. 1985. Inhibition of pokeweed mitogen-induced $\mathrm{B}$ cell differentiation by compounds containing primary amine or hydrazine groups. Clin. Exp. Immunol. 59:69-76.

10. Yu, C.-L., and M. Ziff. 1985. Effects of long-term procainamide therapy on immunoglobulin synthesis. Arthritis Rheum. 28:276-284.

11. Cornacchia, E., J. Golbus, J. Maybaum, J. Strahler, S. Hanash, and B. Richardson. 1988. Hydralazine and procainamide inhibit T cell DNA methylation and induce autoreactivity. J. Immunol. 140:21972200.

12. Tannen, R. H., and W. W. Weber. 1980. Antinuclear antibodies related to acetylator phenotype in mice. J. Pharmacol. Exp. Ther. 213:485-490.

13. Rubin, R. L., J. P. Uetrecht, and J. E. Jones. 1987. Cytotoxicity of oxidative metabolites of procainamide. J. Pharmacol. Exp. Ther. 242:833-841.

14. Uetrecht, J. P., B. J. Sweetman, R. L. Woosley, and J. A. Oates. 1984. Metabolism of procainamide to a hydroxylamine by rat and human hepatic microsomes. Drug Metab. Dispos. 12:77-81.

15. Budinsky, R. A., S. M. Roberts, E. A. Coats, L. Adams, and E. V. Hess. 1987. The formation of procainamide hydroxylamine by rat and human liver microsomes. Drug Metab. Dispos. 15:37-43.

16. Uetrecht, J. P. 1985. Reactivity and possible significance of hydroxylamine and nitroso metabolites of procainamide. J. Pharmacol. Exp. Ther. 232:420-425.

17. Rubin, R. L., J. E. Jones, and J. P. Uetrecht. 1987. Metabolism of procainamide to the reactive hydroxylamine by leukocytes. Fed. Proc. 46:1380a. (Abstr.)

18. Uetrecht, J., N. Zahid, and R. Rubin. 1988. Metabolism of procainamide to a hydroxylamine by human neutrophils and mononuclear leukocytes. Chemical Research in Toxicology. 1:74.

19. Curnutte, J. T., R. Kuver, and P. J. Scott. 1987. Activation of neutrophil NADPH oxidase in a cell-free system. J. Biol. Chem. 262:5563-5569.
20. Parkos, C., M. Dinauer, A. Jesaitis, S. Orkin, and J. Curnutte. 1987. Absence of both the $91 \mathrm{~K}$ and $22 \mathrm{~K}$ subunits of cytochrome b558 in two genetic forms of chronic granulomatous disease. Blood. 70:93a. (Abstr.)

21. Quie, P. G., E. L. Kaplan, A. R. Page, F. L. Gruskay, and S. E. Malawista. 1968. Defective polymorphonuclear-leukocyte function and chronic granulomatous disease in two female children. $N$. Engl. J. Med. 278:976-980.

22. Holmes, B., B. H. Park, S. E. Malawista, P. G. Quie, D. L. Nelson, and R. A. Good. 1970. Chronic granulomatous disease in females. N. Engl. J. Med. 283:217-221.

23. Curnutte, J. T. 1988. Classification of chronic granulomatous disease. In Hematology/Oncology clinics of North America. Phagocytic Defects II: Abnormalities of the respiratory burst. J. T. Curnutte, editor. W. B. Saunders, Philadelphia. 241-252.

24. Skoog, W. A., and W. S. Beck. 1956. Studies on the fibrinogen, dextran, and phytohemagglutnin methods of isolating leukocytes. Blood. 11:436-454.

25. Johnston, Jr., R. B., and J. E. Lehmeyer. 1976. Elaboration of toxic oxygen by-products by neutrophils in a model of immune complex disease. J. Clin. Invest. 57:836-841.

26. Van Epps, D. E., J. G. Bender, J. A. Steinkamp, and D. E. Chenoweth. 1985. Modulation of neutrophil-reduced pyridine nucleotide content following stimulation with phorbol myristate acetate and chemotactic factors. J. Leukocyte Biol. 38:587-601.

27. Fujii, S., and Y. Hitomi. 1981. New synthetic inhibitors of $\mathrm{Clr}, \mathrm{Cl}$ esterase, thrombin, plasmin, kallikrein and trypsin. Biochim. Biophys. Acta. 661:342-345.

28. Bretz, U., and M. Baggiolini. 1974. Biochemical and morphological characterization of azurophil and specific granules of human neutrophilic polymorphonuclear leukocytes. J. Cell Biol. 63:251-269.

29. Brittinger, G., R. Hirschhorn, S. D. Douglas, and G. Weissmann. 1988. Characterization of a hydrolase-rich fraction from human lymphocytes. J. Cell Biol. 37:394-411.

30. Fishman, W. H. 1974. Beta-Glucuronidase. In Methods of enzymatic analysis. H. U. Bergmeyer, editor. Academic Press, Inc., New York/London. 929-932.

31. Klebanoff, S. J., and C. B. Hamon. 1972. Role of myeloperoxidase-mediated antimicrobial systems in intact leuokocytes. J. Reticuloendothel. Soc. 12:170-196.

32. DeChatelet, L. R., C. E. McCall, M. R. Cooper, and P. S. Shirley. 1974. Ascorbic acid levels in phagocytic cells. Proc. Soc. Exp. Biol. Med. 145:1170-1173.

33. Russell, A. S. 1981. Drug-induced autoimmune disease. Clin. Immunol. Allergy. 1:57-76.

34. Hess, E. V., R. J. Donovan-Brand, and L. E. Adams. 1988. In Vitro effects of procainamide metabolites on human cellular function. Arthritis Rheum. 31:S53. (Abstr.)

35. Totoritis, M. C., E. M. Tan, E. M. McNally, and R. L. Rubin. 1988. Association of antibody to histone complex H2A-H2B with symptomatic procainamide-induced lupus. N. Engl. J. Med. 318:1431-1436. 